\title{
Proceedings of the Association of British Neurologists, University of Keele, 15-16 April 1993
}

DETECTION OF CIRCULATING CEREBRAL EMBOLI

H Markus, A Loh, MM Brown. St George's Hospital Medical School, London, UK

Theoretically it should be possible to detect circulating solid cerebral emboli as high intensity signals on a Doppler ultrasound display, and recently such signals have been noted in patients with potential embolic sources.

An in vivo validation study was carried out. Pathological emboli (atheroma, thrombus and platelet aggregates) were introduced into the sheep proximal carotid artery while the ipsilateral distal carotid artery was insonated using a transcranial Doppler ultrasound machine. A total of 74 emboli, with sizes as small as $0.2 \mathrm{~mm}$, were introduced and all were detected as short duration high intensity signals. Smaller emboli could not be made, but microspheres as small as 10 microns were detected.

A logarithmic correlation was found between embolus size and amplitude of signal (thrombus rho $=0.87$, platelet 0.97 , atheroma 0.89 ); the signal associated with platelet emboli was significantly less intense than that with thrombus $(p<0.05)$ or atheroma $(p<0.005)$. A significant linear correlation was found between embolus size and duration of high intensity signal (thrombus $r=0.91$, platelet 0.82 , atheroma $0 \cdot 82)$.

This new technique will allow detection of circulating cerebral emboli in humans. Analysis of signal parameters will give information on the size, and possibly the nature, of such emboli. It may allow selection of patients with particularly high risk of subsequent embolic stroke. Similar signals have been detected in subjects with carotid stenosis and cardiac valvular disease.

COMPARISON OF MAGNETIC RESONANCE ANGIOGRAPHY, INTRA-ARTERIAL DIGITAL SUBTRACTION ANGIOGRAPHY AND DUPLEX ULTRASOUND IN THE ASSESSMENT OF EXTRACRANIAL CAROTID ARTERY STENOSIS GR Young, PRD Humphrey, MDM Shaw, TE Nixon, ETS Smith. The Walton Centre for Neurology and Neurosurgery, Liverpool, UK

The authors carried out a triple blind comparison of duplex ultrasound (US), intraarterial digital subtraction $x$-ray angiography (DSA) and $2 \mathrm{D}$ and $3 \mathrm{D}$ timeof-flight magnetic resonance angiography (MRA) in the assessment of the extracranial internal carotid artery. Patients were screened with US to exclude those with less than $30 \%$ stenosis of the symptomatic carotid artery. A total of 70 consecutive patients referred for angiography, with symptoms of carotid territory ischaemia, had investigation.
Percentage stenosis values were grouped into mild (0-30\%), moderate (31-69\%), severe $(70-99 \%)$ or occluded categories. Analysis of the first 36 patients is complete.

Spearman's rank correlations were 0.92 (MRA and DSA), 0.90 (MRA and US) and 0.93 (DSA and US).

For clinically important differences the chance corrected proportional agreement or Kappa (K) statistic was used, where $\kappa=0.61-0.80$ indicates good agreement and $\kappa=0.81-1.00$ indicates very good agreement. $\kappa=0.68$ (MRA v DSA), 0.68 (DSA v US) and 0.70 (MRA v US) Weighted kappa values were $\kappa_{\mathrm{w}}=0.80,0.80$, and 0.81 respectively.

Assuming DSA to indicate the true situation (data will be presented to question this) the sensitivity and specificity for diagnosing $70-99 \%$ stenosis by MRA were $85 \%$ and $96 \%$ respectively, with positive predictive value $92 \%$ and negative predictive value $91 \%$.

Investigation with MRA together with US will largely replace the need for DSA when assessing suitability for prophylactic carotid endarterectomy.

COAGULATION ABNORMALITIES IN BENIGN INTRACRANIAL HYPERTENSION

JD Sussman, GAB Davies-Jones, $M$ Greaves, $M$ Leach. Royal Hallamshire Hospital, Sheffield, UK

Study of the pathophysiology of benign intracranial hypertension (BIH) suggests that either an increased resistance to CSF flow across the arachnoid villi, or an increased pressure within the venous system may give rise to raised intracranial pressure. The similarity between the symptoms and signs which arise in venous sinus thromboses and $\mathrm{BIH}$ raises the possibility that microvascular occlusion which is not visible on conventional angiography might give rise to cases of BIH.

Thirty eight patients with BIH were therefore investigated by measurement of blood cell counts, fibrinogen levels, anticardiolipin antibodies, lupus anticoagulant autoantibodies, protein $\mathrm{C}$ and $\mathrm{S}$, and antithrombin III levels.

Twenty three $(60 \%)$ showed abnormalities previously associated with thrombotic tendencies. Twelve (32\%) of the 38 had lupus anticoagulants, ten $(26 \%)$ had abnormally high fibrinogen levels. Some other rare causes of hypercoagulability were found.

These findings suggest that, as suspected, $\mathrm{BIH}$ is a symptom complex with a variety of causes and a new approach to treatment is suggested.

A STUDY OF HEREDITARY ESSENTIAL TREMOR PG Bain, LJ Findley, CD Marsden. MRC Human Movement and Balance Unit, London UK
Objective: To determine the phenotype of hereditary essential tremor (HET).

Background: Clinical information on HET is scarce compared to that on "essential tremor"

Methods: 20 index cases with incontrovertible HET and their kindreds were studied (51 of whom were affected).

Results: HET is autosomal dominant, with bimodal age of onset (median: 17 years). Penetrance was virtually complete by the age of 65 . The segregation ratio for affected/total kin was $\mathbf{0 . 4 8}$. There were no examples of the disease skipping a generation. Men and women were equally affected. The typical phenotype was a mild symmetrical action tremor of the upper limbs. No isolated tremors of the legs, head, face, voice, jaw and tongue were encountered and no dystonic postures occurred. There was a strong association with classical migraine but none with Parkinson's disease. Primary writing tremor and primary orthostatic tremor did not occur. A total of $70 \%$ of head tremor was of a "no-no" variety. Fifty percent of cases were alcohol responsive but in $20 \%$ of families heterogeneity of responsiveness existed. Tremor severity and disability increased with age and the duration of tremor but age at onset had no predictive value on outcome.

Conclusion: Hereditary essential tremor does not produce dystonia and differs in many respects from previous descriptions of "essential tremor".

HEMISPHERECTOMY; AN EFFECTIVE TREATMENT FOR RASMUSSEN'S SYNDROME Z Matkovic, JM Oxbury, CBT Adams, SM Oxbury, J Morris, Z Zaiwalla. The Radcliffe Infirmary, and the Park Hospital, Oxford, UK

Seven patients with Rasmussen's syndrome had hemispherectomy ( 7 hemispherectomy, 1 restricted corticectomy). Pathology confirmed cortical damage (7), and inflammation (5). Pre-operative features were: all had onset with seizures (tonic/clonic 4, simple focal 2 , complex focal 1) at age 4-8 years; unilateral weakness developed $0 \cdot 4-6$ years later; 2 had dysphasia and 2 hemianopia. Electroencephalography showed excess slow activity bilaterally (7), spikes (unilateral 5, bilateral-2). Neuroimaging showed unilateral cerebral atrophy (6). CSF examination revealed pleocytosis (1), oligoclonal banding (1). IQ ranged 54-105 (median 67). Clinical deterioration developed over months (5) or years (2). Control of seizures was not achieved by: anticonvulsant drugs (7), restricted corticectomy (5), ketogenic diet (2), steroids (2), gamma globulin (2). Hemispherectomy was performed 0.5-20 years after onset (median, 3). Postoperative follow up to 6 years has been with regular clinical and neuropsychological assessment. After a follow up period of at least 3 
months, six were walking independently, one with assistance and five were seizure free. Psychosocial functioning was assessed by questionnaire. Parents have all described hemispherectomy as "successful" and outcome as "very satisfactory".

It is concluded that hemispherectomy is the only satisfactory treatment for Rasmussen's syndrome.

EFFECT OF STRUCTURED BACKGROUNDS ON SMOOTH PURSUTT EYE MOVEMENTS

MC Lawden, H Bagelmann, TJ Crawford, TD Matthews, C Kennard. Charing Cross and Westminster Medical School, London, UK

The oculomotor smooth pursuit system is driven by the slip of the target image upon the retina arising from errors in matching eye and target velocities. Pursuit of an object moving against a structured background will result in retinal flow in the direction opposite to target movement. Central mechanisms allow these distracting signals to be overridden effortlessly. To isolate the anatomical substrate of this capacity the effect of the presence of a structured background upon smooth pursuit in 27 patients with focal cerebral lesions was studied. Studies on normal subjects confirmed that a background has little effect upon pursuit.

Eye movements were recorded by infrared oculography or the scleral search coil method. The target was a bright spot moving horizontally in a triangular waveform of amplitude $22.5 \mathrm{deg}$ visual angle, at either $10,20,30$, or $36.5 \mathrm{deg} / \mathrm{s}$. Data were collected in darkness and with a structured background. Fourteen patients showed a significant reduction of gain with a structured background, while the remaining 13 showed little or no effect. Comparison of the location of the cerebral lesions in these two groups suggested that lesions in the inferior parietal cortex (area 40) result in disruption of pursuit in the presence of a background.

ANTI-GANGLIOSIDE ANTIBODIES IN PERIPHERAL NEUROPATHY

HJ Willison, J Veitch, G Paterson, PGE Kennedy. University of Glasgow, UK

Autoantibodies reactive with polysialylated gangliosides are becoming increasingly identified in patients with acute and chronic peripheral neuropathies.

In Miller Fisher syndrome (MFS), it has been observed that all (4/4) consecutive cases that have recently been seen had antiGQ1b IgG autoantibodies with titres ranging from $1 / 960$ to $1 / 13500$ and lower titre IgM anti-GQ1b antibodies. These antibodies are absent in controls. 3/4 MFS cases also showed reactivity with GD1b, GD3 and GDla, mainly in the IgG fraction. These data concur with 2 other recent reports of similar findings.

A patient has also recently been identified with a chronic sensory demyelinating neuropathy and an IgM paraproteinaemia in whom the paraprotein reacts at titres in excess of $1 / 100,000$ with NeuNAc(a2-8)NeuNAc(a2-3)Gal configured disialosyl groups present on the gangliosides GD1b, GT1b, GQ1b and GD3. This patient is similar to 4 other reported cases.
These data indicate that clinical phenotypes can be segregated serologically on the basis of their pattern of anti-ganglioside antibody reactivities in that a) anti-GQ1b IgG antibodies appear to be a highly specific and diagnostically useful marker for MFS and b) a large fibre predominantly sensory demyelinating neuropathy is particularly associated with IgM anti-disialosyl antibodies.

NERVE GROWTH FACTOR IN INJURED HUMAN PERIPHERAL NERVE: IMPLICATIONS FOR NERVE REPAIR

P Anand, R Birch, P Foley, D Sinicropi, S Peroutka. The Royal London Hospital Medical College, London, the Royal National Orthopaedic Hospital, Stanmore, UK and Genentech Inc, San Francisco, USA

Animal models show that degenerative changes in injured nerves, including decreased expression of sensory neuropeptides and neurofilaments, and axonal atrophy, may be caused by deprivation of nerve growth factor (NGF), and can be reversed by administration of exogenous NGF. The imminent availability of recombinant human NGF for clinical use led to this study of brachial plexus injury in obstetric and road traffic accident cases, where despite advances in reconstructive surgery, nerve degeneration and failure of regeneration result in chronic pain and poor recovery. NGF concentrations in sural nerves used for grafting in these cases ranged between 2,000 and $6,000 \mathrm{pg} / \mathrm{g}$, in accord with previously reported levels of $2,400 \pm$ $500 \mathrm{pg} / \mathrm{g}$ in post-mortem adult human sciatic nerve. The levels of NGF were lower $(500-2,000 \mathrm{pg} / \mathrm{g})$ in avulsed spinal roots of both adults and neonates 3 months postinjury. NGF levels were usually low but variable in different cases just proximal and distal to the site of injury, and in dorsal root ganglia related to avulsed roots: this may partly explain the variable spontaneous and post-surgical outcomes. As NGF concentrations in dorsal column and dorsal horn of post-mortem human spinal cord range between 10,000 and $20,000 \mathrm{pg} / \mathrm{g}$, local exogenous NGF administration appears indicated in re-implantation of avulsed dorsal roots and peripheral nerve repair.

MOLECULAR STUDIES IN SPINAL MUSCULAR ATROPHY

KE Morrison, RJ Daniels, MJ Francis, KE Davies. Institute of Molecular Medicine, University of Oxford, UK

Childhood onset proximal spinal muscular atrophy (SMA) is a neuromuscular disease characterised by selective loss of alpha motor neuron cell bodies. Three forms have been distinguished on the basis of severity of disability and age at onset. All three show autosomal recessive inheritance and have been mapped by linkage analysis to probes at $5 q 11 \cdot 2-13 \cdot 3$.

We have expanded the original linkage by genotyping over 60 SMA pedigrees with many novel microsatellites generated from yeast artificial chromosomes (YACs) and cosmids which map within the candidate SMA interval. Multipoint mapping and analysis of key recombinants has refined the disease locus to a $1 \mathrm{cM}$ region, flanked by markers D5S345 and 2a9.1. The novel microsatellites that have been developed are now being used in several laboratories worldwide to provide more accurate prenatal prediction of SMA.

YACs have been isolated for all relevant markers and by generating end clones and subsequent YAC walking, the entire candidate region is now almost completely cloned. Genes from this region are being isolated by directly screening fetal brain and spinal cord cDNA libraries with YACs and cosmid contigs. One expressed sequence characterised to date is of particular interest, as it shows homology to a portion of the androgen receptor, the first exon of which shows a trinucleotide expansion in spinal bulbar muscular atrophy.

SURGICAL TREATMENT OF NEUROGENIC

THORACIC OUTLET SYNDROME: A FOLLOW UP STUDY

Z Matkovic, M Donaghy, PJ Morris. Radcliffe Infirmary, and John Radcliffe Hospital, Oxford, UK

Twenty eight patients ( $23 \mathrm{f}, 5 \mathrm{~m}$; age 23-62 years) were assessed from 3 months to 16 years following 23 operations for suspected neurogenic thoracic outlet syndrome.

Cervical ribs were demonstrated by plain x-rays (15) or MRI only (1). Cervical rib resection (17) produced resolution (9) or significant improvement of symptoms (7). Symptoms continued to progress in one patient subsequently diagnosed as suffering from syringomyelia.

Twelve patients had features of thoracic outlet syndrome but no cervical ribs. In 4 no definite operative abnormality was found, scalenus anterior was divided, and symptoms improved in all 4. Brachial plexus was decompressed by first rib resection in 7. Symptoms resolved in 2, improved in 2 , resolved initially and after 3 repeat operations for recurrences (due to regrowth of first rib, development of a fibrous band, and fibrous tissue formation respectively) in 1 , and progressed in 2 in whom chronic spinal muscular atrophy was diagnosed years later. Symptoms improved in one patient following C7 transvex process resection.

Following surgery, pain (22/24 patients) and sensory disturbance (21/25 patients) diminished, and muscle power improved (10/21 patients). Surgical complications were transient: shoulder girdle pains (3), phrenic nerve palsy (2), neurological worsening (1).

It is concluded that surgical treatment of neurogenic thoracic outlet syndrome is effective and well-tolerated both in patients with and without a cervical rib.

\section{HOW SHOULD WE INVESTIGATE PATIENTS}

WITH SUSPECTED DEFECTS OF THE

MITOCHONDRIAL RESPIRATORY CHAIN?

MJ Jackson, LA Bindoff, DM Turnbull. University of Newcastle upon Tyne, UK

Respiratory chain disease (RCD) is an important cause of neurological illness. RCD may present in a variety of ways and is therefore considered in many differential diagnoses. Detailed evaluation of RCD is difficult, time-consuming and is only undertaken in specialist units. Since it is not possible to investigate every patient in whom RCD is suspected in such detail, can patients be selected for investigation by routine clinical tests? 
The authors evaluated the results of serum and CSF lactate, EMG, serum creatine kinase activity (CK) and muscle histology in 50 patients with RCD (established by biochemical and molecular analysis).

Serum lactate was found helpful but was not sufficiently sensitive for screening purposes in certain groups of RCD, for example, myopathy, elevated in all patients with Fanconi/myopathy syndrome and of no value in progressive external ophthalmoplegia. CSF lactate was consistently elevated in RCD that presented with CNS involvement. CK and EMG were normal or near normal in RCD even in patients with myopathies, and the finding of unremarkable CK and EMG in a myopathy is an important clue to metabolic muscle disease.

Muscle histology does not always show ragged-red fibres, even in patients with RCD myopathy. We have found cytochrome oxidase activity a better indicator of RCD.

The data have been used to propose an algorithm for the evaluation of RCD.

PROTON SPECTROSCOPY IN DEMYELINATING LESIONS

CA Davie, CP Hawkins, WI McDonald, DH Miller. Institute of Neurology, London, UK

Magnetic resonance spectroscopy (MRS) is providing useful information about chemical pathology in an increasing number of neurological diseases. In multiple sclerosis consistent abnormalities have been reported using this technique. N-acetyl Aspartate, a marker of neuronal impairment and loss, is decreased within MS plaques while Choline and inositol show an increase in concentration probably due to increased membrane turnover. Using short echo time proton spectroscopy of $10 \mathrm{msecs}$, six patients were followed at monthly intervals with symptomatic or MRI evidence of acute demyelinating lesions which displayed Gadolinium enhancement.

Spectroscopy of these lesions showed an increase in the Choline/Creatine ratio, an increase in the Inositol/Creatine ratio and a decrease in the NAA/Cr. ratio compared to healthy age matched controls and normal appearing white matter (NAWM). In addition, spectra from the enhancing lesions showed large peaks relative to NAWM and healthy controls at 0.9 and $1.3 \mathrm{ppm}$ which are likely to represent myelin breakdown products. Spectra from chronic plaques showed much smaller peaks in these regions similar to NAWM.

MRS allows the in vivo detection of myelin breakdown products in MS. This may prove useful in differential diagnosis and in monitoring patients involved in future treatment trials.

HOW NEURONS INFLUENCE THE PATHWAY OF OLIGODENDROCYTE PROGENITOR DEVELOPMENT

JP Zajicek, DAS Compston. University of Cambridge, UK

For efficient myelination to occur in the CNS oligodendrocyte progenitors must proliferate, migrate and differentiate in an ordered sequence. To investigate these processes in detail, the authors adopted an in vitro tissue culture model of myelination using purified embryonic rat dorsal root ganglia upon which are plated purified oligodendrocyte progenitor cells. Analysis of these cultures has demonstrated an axonal signal which stimulates mitosis in progenitors and this is mediated by platelet derived growth factor (PDGF). Secondly, there is an alteration of cell surface molecules on progenitors in co-cultures with axons so that ganglioside expression is modified. Thirdly, there is a bias of the differentiation pathway seen in progenitors grown in serum free medium which restricts the production of oligodendrocytes and promotes differentiation into astrocytes. These findings are relevant both to normal development of structures at the node of Ranvier and may explain the nature of astrocytosis which characterises chronic demyelination.

QUANTITATIVE MRI IN PATIENTS WITH

CLINICALLY ISOLATED SYNDROMES

SUGGESTIVE OF MS

M Filippi, MA Horsfield, WI McDonald, DH Miller. Institute of Neurology, London, UK and Scientific Institute S. Raffaele, Milan, Italy.

A semi-automated quantitative measurement of brain MRI abnormalities seen at presentation and at five year follow up was performed in 84 patients presenting with a clinically isolated syndrome (CIS) of the optic nerves, brainstem or spinal cord suggestive of multiple sclerosis (MS).

Patients who developed MS during follow up had a higher lesion load at presentation compared to those who did not $(p<$ 0.0001 ). There was a strong correlation of the MRI lesion load at presentation with both the increase in lesion load in the next five years $(p<0.0001)$ and disability at follow up $(p<0.0001)$. At follow up, disability and brain lesion load were strongly correlated in patients who developed MS (p $=0.0002$ ).

These results establish that MRI at presentation with CIS suggestive of MS is useful in predicting the subsequent clinical course, and the development of new MRI lesions. This suggests that quantitative brain MRI will be helpful in selecting patients with early clinical MS for treatment trials, and for subsequent monitoring of their response to treatment.

NERVE GROWTH FACTOR IN HUMAN SPINAL CORD: CHANGES IN MOTOR NEURON DISEASE P Anand, P Foley, J Martin, M Swash, D Sinicropi, S Peroutka. London Hospital Medical College, London, UK, and Genetech Inc, San Francisco, USA

Classical studies have shown that nerve growth factor (NGF) is selectively trophic to peripheral sympathetic and sensory fibres and central cholinergic neurons. Using an assay based on recombinant human NGF, regional NGF levels were measured in postmortem human spinal cord from patients with motor neuron disease (MND) and matched controls. Our findings extend the classical view of NGF. High concentrations of NGF were found in normal dorsal column, dorsal horn, dorsolateral fasciculus and anterior white matter (range 10 to 20 $\mathrm{ng} / \mathrm{g}$ ), which are several fold higher than in peripheral nerve, and include regions not thought to be dependent on NGF. As cervical ventral horn provides limited tissue, studies are in progress to measure NGF in this region. There was a significant decrease of NGF in dorsal column and dorsal horn in MND cases, while an increase was found in dorsolateral fasciculus. The low NGF levels in dorsal cord may result partly from decreased uptake and transport from atrophied peripheral tissues, and perhaps relate to the sensory abnormalities documented in MND. As there was evidence of corticospinal tract degeneration in the dorsolateral fasciculus in the MND specimens, reactive glial cells may be the source of the increased NGF in this region. It is proposed that NGF may play different primary and secondary roles in neurodegeneration, the latter akin to its role in inflammation.

THE SIGNIFICANCE OF THE CHANGE IN THE PROTON NMR SPECTRUM IN EAE

RE Brenner, PMG Munro, SCR Williams, GJ Barker, CP Hawkins, WI McDonald. Institute of Neurology, London, UK

Proton magnetic resonance spectroscopy (MRS) provides data about the relative concentrations of compounds from chosen volumes down to $1 \mathrm{~cm}^{3}$ (the size of many MS lesions). Acute lesions show an elevation of the choline:creatine ratio, and some chronic lesions show a decrease in $\mathrm{N}$-acetylaspartate (NAA). The former has been attributed to demyelination and the latter to neuronal loss, but conclusive evidence is lacking.

The evolution of the spectra and histological appearances in $\mathbf{4 0}$ guinea pigs with acute experimental allergic encephalomyelitis was studied (EAE) and compared with 26 controls. The increase in choline correlated not with demyelination but with inflammation, and the reduction in NAA was not accompanied by neuronal loss; it is likely that it results from neuronal dysfunction. These findings have important implications for the interpretation of MRS in patients.

THE CONSEQUENCES OF "FRONTAL" IMPAIRMENT FOR REACTION TIMES IN PARKINSON'S DISEASE

JE Harrison, S Goodrich, C Kennard, L Henderson. Charing Cross \& Westminster Medical School, London, UK

Studies of normal subjects and patients with Parkinson's disease (PD) have converged on the idea that the processes underlying choice and simple reaction time (RT) are dissociable. In this regard, a key finding has been a selective impairment of simple RT in patients with PD. Unfortunately, recent studies have not been able to reliably replicate this phenomenon.

The authors present an experiment in support of the selective impairment of simple RT, showing it to be evident only in a sub-population of PD patients. All PD patients in this experiment were tested on the Wisconsin Card Sorting Test, a neuropsychological test held to be sensitive to frontal lobe dysfunction. According to performance, patients were allocated to either a group showing signs of frontal dysfunction $(n=9)$, or a group $(n=9)$ showing no frontal impairment.

A comparison of mean reaction times for PD patients with evidence of frontal dysfunction, and those without, showed a significant interaction demonstrating "frontal" PDs to be significantly slower in simple RT, but not reliably so in choice RT 
$(p<0.01)$. The working hypothesis is that these patients suffer from a frontal-type syndrome which disrupts the preparatory process essential in normal simple RT performance.

FAMILIAL ALZHEIMER'S DISEASE: THE NEUROLOGY OF APP MUTATIONS AM Kennedy, SN Newman, J Ball, $P$ Roques, EK Warrington, RSJ Frackowiak, MN Rossor. St Mary's Hospital, MRC Cyclotron Unit, and The National Hospital for Neurology and Neurosurgery, London, UK

The description of four mutations in the amyloid precursor protein gene co-segregating with the affected individuals with Familial Alzheimer's disease provides evidence for allelic heterogeneity in this condition. In order to assess for phenotypic heterogeneity, the 9 living affected individuals from the three British pedigrees $\left(\mathrm{F}-1_{\text {(val-gly) }}, \mathrm{F}-2_{\text {(val-ile) }}\right.$ and $\left.\mathrm{F}-3_{\text {(val-ile) }}\right)$ were assessed and the case records of the other deceased individuals reviewed. Four individuals were very severely affected. The mean age at onset for each family was 52, 55 and 52 years respectively. All individuals presented with cognitive impairment but with little insight into their cognitive difficulties. Myoclonic jerks were observed in all living individuals and seizures were a frequent manifestation. (5 out of 10 cases in APP-3). Neuropsychological testing in 4 cases showed severe global memory deficits and widespread generalised cognitive decline with preservation of object picture naming skills. Positron Emission Tomography demonstrated bi parietal bitemporal hypometabolism with additional frontal hypometabolism proportional to the severity of dementia.

Despite the severity of dementia in the affected cases studied, similar ages at onset, clinical and neuropsychological profiles are apparent.

NEW APPROACHES TO DIAGNOSIS IN PRION DISEASES

J Collinge, MS Palmer. St Mary's Hospital Medical School, London, UK.

The transmissible spongiform encephalopathies are now known to have a larger range of phenotypic expression than was hitherto realised and these conditions, which include cases in which the classical pathological features of spongiform encephalopathies are absent, may now be classified as prion diseases. The prion protein $(\mathrm{PrP})$ and its gene play a central role in the aetiology of inherited, acquired and sporadic prion diseases. The availability of both prion protein gene analysis and the detection of the disease related isoform of prion protein by immunoblotting has enabled the re-classification of a number of atypical neurodegenerative cases as variants of prion disease. We have screened DNA from over 700 cases and controls for PrP mutations and detected over 20 cases with pathogenic mutations. Immunoblotting has been applied on cases where frozen tissue is available. The authors here previously reported that normal polymorphisms of the PrP gene can affect both disease susceptibility and phenotype. We have recently identified 9 individuals with deletions in the PrP gene.
These deletions constitute a new class of PrP polymorphisms and appear, to date, to be benign. In several of the cases in which a pathogenic mutation was detected a family history of neurodegenerative disease was not initially apparent. PrP gene analysis should be considered in all cases of presenile dementia. In addition we are developing a bioassay for infectivity of brain tissue using transgenic mice. This combination of DNA analysis, immunoblotting and transgenic bioassay should allow greater understanding of this group of conditions and provide a useful adjunct to traditional clinicopathological diagnostic criteria.

IS READING A SPARED ABILITY IN DEMENTIA OF ALZHEIMER'S TYPE (DAT)?

J $R$ Hodges, $N$ Graham, $K$ Patterson. University of Cambridge and MRC Applied Psychology Unit, UK

It is often assumed that both whole-word (lexical) and sound-based (phonological) reading are preserved in DAT. Exception word pronunciation (for example, pint, island, naive) is used as a measure of premorbid intelligence in the National Adult Reading Test (NART). Yet it is well established that semantic memory is impaired in DAT which would be expected to produce selective impairment in exception word reading (that is, surface dyslexia). To date, studies of reading in DAT are limited and have yielded conflicting results.

The authors assessed reading in 45 patients with DAT and 25 controls on 252 regular and exception words in three matched frequency bands, and the NART. The DAT cases were divided into three groups according to performance on the Mini-Mental State Examination (MMSE)-minimal ( $>23$ ); mild (15-23); and moderate $(<15)$-matched for age and education. Analysis of variance showed that there were significant effects of regularity, word frequency and disease severity as well as a significant group $\mathrm{X}$ word type interaction (that is, a pattern of surface dyslexia). Impairment in exception, but not regular word, reading was highly correlated with measures of semantic memory (for example, naming, picture-pointing etc.). Furthermore, there was a significant drop in performance on the NART with increasing disease severity which would lead to an erroneous underestimation of IQ by 15 points ( $1 \mathrm{SD}$ ) in the moderate DAT group.

In conclusion, reading of exception words is not spared in DAT; as predicted, features of surface dyslexia are present. This finding casts doubt upon the reliability of the NART in predicting premorbid IQ in DAT.

CORTICAL VERSUS SUB-CORTICAL DEMENTIA: A REAL DISTINCTION?

A Rosser, N Graham, JR Hodges. University of Cambridge, UK

The distinction between cortical and subcortical dementia is disputed. Although there is growing evidence that aetiologically distinct forms of dementing illness produce qualitatively distinct patterns of cognitive deficit, most previous comparative studies have been methodologically flawed. The authors compared two patient groups with predominantly subcortical disorders-progressive supranuclear palsy (PSP, $n=10$ ) and Huntington's disease (HD, $n=10)$ and patients with the prototypic form of cortical disease, dementia of Alzheimer's type (DAT, $\mathrm{n}=10$ ), matched for overall level of dementia on the Mini-Mental State Examination (MMSE) and Dementia Rating Scale (DRS) on a comprehensive neuropsychological test battery. In no patient was the degree of dementia severe (MMSE range 18-30). Performance on tests of naming, word-picture matching, language comprehension and non-verbal semantic knowledge was similar across the groups. Patients with PSP and HD were significantly more impaired on tests of visuo-spatial ability and initiation; $A D$ patients were more impaired on tests of verbal episodic memory. Verbal fluency was impaired in all patient groups compared to controls, but letter fluency was relatively more impaired than category fluency in the PSP and HD groups. A battery of tests designed to assess semantic knowledge about the same items, within and across modalities, was given; performance was similar in the three patient groups, although the nature of the deficit underlying the impairment in the groups appears to be different. The results offer support for the cortical-subcortical distinction.

SUBCLINICAL INVOLVEMENT OF THE NERVOUS SYSTEM IN BEHÇET'S DISEASE A NEUROPSYCHOLOGICAL STUDY

F Ahmed, J Bamford, A Coughlan, MA Chamberlain, B Noble. St James's University Hospital and The General Infirmary, Leeds, UK.

A third of patients with Behçet's disease (BD) will develop neurological symptoms (neuro-BD). They have a bad prognosis and the risks of more aggressive chemotherapy are considered justifiable. However, the first "clinical" event may leave severe neurological sequelae and methods of identifying patients with neuro-BD at an early "subclinical" stage are required. MRI has shown "asymptomatic" cerebral lesions but remains scarce in the UK and there is insufficient evidence to justify regular scanning. Simple neuropsychological assessments have been used to detect subtle deficits in conditions such as MS and would be a practical alternative if they predicted the development of neuro-BD. We report here the results of the initial assessment of a cohort of patients with BD who will be followed prospectively.

We assessed 21 patients who fulfilled the International Study Group (ISG) criteria for $B D$ and age, sex and IQ matched healthy controls. None had symptoms or signs on routine examination to suggest current or previous involvement of the nervous system. Their disease activity was graded according to ISG recommendations.

Although patients were more anxious $(p<0.02)$ and depressed $(p<0.01)$ than controls there was no correlation between mood and performance on other tests. Significant impairment $(p<0.05)$ in patients with $\mathrm{BD}$ was found on the tests of alertness, verbal and non-verbal memory, and naming. There was no correlation between the systemic disease activity and the extent of neuropsychological impairment. 
TREMOR ASSESSMENT IN CLINICAL TRIALS PG Bain, LJ Findley, $\mathbf{P}$ Atchison, $M$ Behari, M Vidailhet, JC Rothwell. MRC Human Movement \& Balance Unit, London, UK

We developed a clinical rating scale for measuring the severity of essential tremor at specific anatomical sites. Then using 4 raters and 20 patients with essential tremor, the rating scale was assessed for both interand intra-rater reliability. The scale was also validated against a disability selfquestionnaire, accelerometry and the degree of impairment found in patients' handwriting and spirography.

The rating scale proved to have substantial inter- and intra-rater reliability (assessed using Cohen's Kappa coefficients). Furthermore, the clinically rated scores for the patients' right upper limb postural tremors correlated well with the results obtained from the disability self-questionnaires and assessments of spirography and handwriting impairments (all significant at $\mathrm{p}<0.01$ ).

The results of upper limb accelerometry had no relationship with either disability or spirography and writing impairment but did correlate with the raters' scores for upper limb postural tremor $(p<0.01)$.

It is concluded that: 1) accelerometry does not provide a valid index of tremor induced disability; 2) a clinical rating scale can be reliable and is valid; 3) no other tremor rating scale has been assessed in this way.

BIOENERGETICS OF THE GASTROCNEMIUS MUSCLE IN MYOTONIC DYSTROPHY

PRJ Barnes, GJ Kemp, DJ Taylor, GK Radda. University of Oxford and MRC Clinical and Biochemical Magnetic Resonance Unit, UK

The authors have used ${ }^{31} \mathrm{P}$ magnetic resonance spectroscopy to study bioenergetics in the gastrocnemius muscle of 14 patients with myotonic dystrophy (MyD). At rest, intracellular $\mathrm{pH}$ was higher in the patient group [7.05 \pm 0.01 [SE] v 7.03 $\pm 0.01 ; p=$ 0.009 , Mann-Whitney U statistic]. The intracellular phosphocreatine (PCr) concentration was normal, but that of inorganic phosphate $(\mathrm{Pi})$ was raised at $5.9 \pm 1.1 \mathrm{mM}$ (control $3.6 \pm 0.2 ; p=0.004$ ) and the calculated concentration of free ADP was also raised (33 $\pm 6 \mu \mathrm{M}$ vs $20 \pm 2 \mu \mathrm{M} ; \mathrm{p}=0.04)$. During plantarflexion exercise the patient group exercised for a mean of $5 \cdot 2 \pm 1 \cdot 1$ minutes compared with $10.7 \pm 0.4$ minutes in controls $(p=0.0004)$. The levels of $\mathrm{pH}$, $\mathrm{PCr}, \mathrm{Pi}$ and $\mathrm{ADP}$ at the end of exercise were not different in the two groups, however, and the relationship of $\mathrm{pH}$ and $\mathrm{PCr}$ utilisation was normal. The recovery half times for $\mathrm{PCr}, \mathrm{Pi}$ and $\mathrm{ADP}$ and the apparent calculated mitochondrial $V_{\max }$ for ATP resynthesis following exercise were all normal. These data contrast with those from the forearm flexor in patients with MyD where resting muscle showed normal $\mathrm{pH}$ but there was an abnormal relationship between $\mathrm{pH}$ and $\mathrm{PCr}$ utilisation during exercise. One explanation is that changes in resting $\mathrm{pH}$ are related to regeneration not present within more severely affected muscle.
THE RELATIONSHIP BETWEEN GdENHANCEMENT AND PROTON NMR SPECTRAL CHANGES IN EAE

RE Brenner, SP Morrissey, EPGH Du Boulay, PMG Munro, SCR Williams, GJ Barker, CP Hawkins, WI McDonald. Institute of Neurology, London, UK

The earliest detectable event in the development of the new lesion in MS, as in experimental allergic encephalomyelitis (EAE), is a breakdown in the blood-brain barrier in association with inflammation. The precise sequence of events is however uncertain. We have attempted to elucidate them in acute EAE in guinea pigs. In acute EAE as in MS magnetic resonance spectroscopy (MRS) there is an elevated choline:creatine ratio which at least in the former is associated with inflammation, reflecting increased metabolic turnover in the cellular infiltrate. In sequential studies in 14 animals with EAE compared with 8 controls, we observed an increase in choline:creatine before the onset of Gd-enhancement. This suggests that perivenous inflammation precedes blood-brain barrier breakdown to solutes. Whether the same is true in MS awaits the results of serial MRS studies in patients with much ongoing disease activity.

IMPROVING THE RECORDING OF CLINICAL ASSESSMENT OF STROKE PATIENTS WITH A CLERKING PROFORMA

RJ Davenport, MS Dennis. Western General Hospital, Edinburgh, UK

A thorough clinical assessment is essential for the accurate diagnosis, treatment, rehabilitation, prediction of outcome and secondary prevention in patients with acute stroke. The aims of this study were to measure the completeness of patients' assessments by junior doctors and to determine whether the introduction of a clerking proforma would improve this.

The authors prospectively identified all patients admitted with an acute stroke to the Unit over an 8 month period. Using a carefully constructed form and explicitly worded standards two observers (RJD and MSD) audited the completeness of the recording of the patients' assessments in the medical records. Disappointing results (see table) led to the introduction of a stroke clerking proforma which was followed by a further audit. Although the inter rater reliability of our audit form was reasonable, equal proportions of the records were assessed by each observer before and after the introduction of the proforma to minimise bias. The numbers audited after the introduction of the proforma were based on simple power calculations.

Our results (see table) show that a clerking proforma improves the completeness of recording of patients' assessments and facilitates further audit which may improve patient care.

\begin{tabular}{|c|c|c|c|c|c|}
\hline \multirow{3}{*}{$\begin{array}{l}\text { Examples of Audit Items } \\
\text { Symptoms from witness } \\
\text { Peripheral vascular disease } \\
\text { Cervical Bruit } \\
\text { Conscious level } \\
\text { Swallowing } \\
\text { Driving }\end{array}$} & \multirow{2}{*}{\multicolumn{2}{|c|}{$\begin{array}{l}\text { Before }(n=160) \\
\text { Recorded/ } \\
\text { Standard \% }\end{array}$}} & \multirow{2}{*}{\multicolumn{2}{|c|}{$\begin{array}{l}\text { After }(n=66) \\
\text { Recorded/ } \\
\text { Standard \% }\end{array}$}} & \multirow{3}{*}{$\begin{array}{l}\text { Difference of } \\
\text { Proportion } \\
\text { (with } 95 \% \\
\text { confidence intervals) } \\
6 \%(-16,4 \%) \\
76 \%(68,85 \%) \\
50 \%(41,57 \%) \\
34 \%(27,42 \%) \\
70 \%(60,79 \%) \\
82 \%(73,92 \%)\end{array}$} \\
\hline & & & & & \\
\hline & $\begin{array}{c}88 / 100 \\
23 / 144 \\
79 / 160 \\
105 / 160 \\
32 / 122 \\
7 / 92\end{array}$ & $\begin{array}{r}88 \% \\
16 \% \\
49 \% \\
66 \% \\
26 \% \\
8 \%\end{array}$ & $\begin{array}{l}31 / 33 \\
60 / 65 \\
65 / 66 \\
66 / 66 \\
63 / 66 \\
55 / 61\end{array}$ & $\begin{array}{r}94 \% \\
92 \% \\
99 \% \\
100 \% \\
96 \% \\
90 \%\end{array}$ & \\
\hline
\end{tabular}

THE REFERRED TRIGEMINAL SYNDROME: A POTENTIALLY IMPORTANT AETIOLOGICAL CONCEPT CONCERNING THE GENESIS OF HEADACHE

CJF Davis. The Radcliffe Infirmary, Oxford, UK

Headache is by far the commonest single disorder managed by the neurologist $(30 \%$ of outpatients). A very large part of diagnosis is descriptive rather than aetiologically based. The headaches of many patients fail to fit either with traditional descriptive categories or with the new International Headache Society classification.

A series of sixty outpatients (Age 14-72 mean 39.4; Female:Male $=3: 1$ ) with constantly lateralised anterior head pain is presented. The pain was of 0.5 to 252 months duration (mean 24.6 months), located orbitally in $73 \%$, right sided in $60 \%$, intermittent or persistent equally often, and associated with a relevant cervical history in $54 \%$ or ipsilateral suboccipital tenderness in at least $55 \%$. Of these a small number are discussed in detail in particular respect of features which strongly imply that their pain was triggered from structures at the craniocervical junction without any complaint of pain localised to this area. There is limited but indicative literature to suggest that potentially painful processes localised to this area may, no doubt due to some interaction with the spinal nucleus of the ipsilateral fifth cranial nerve, generate referred pain which is experienced entirely within its "cutaneous" territory. From this, it is speculated that many or all of the larger group of fifty eight patients with no overt or identifiable cause for their symptoms may also have their pain triggered from the craniocervical junction. If this conclusion be correct, it may have wider implications in respect of the mechanism of genesis of headache of a less clearly localised nature.

MRI OF PATIENTS WITH ISOLATED

IDIOPATHIC RETINAL VASCULITIS

A Gass, E Graham, IF Moseley, WI McDonald, DH Miller. Institute of Neurology, London, UK

Retinal involvement (for example, venous sheathing, leakage on fluorescein angiography) in MS patients occurs in up to $40 \%$, whereas only $10 \%$ of patients with retinal vasculitis go on to develop MS. We therefore performed cranial MRI in IRV patients to look for further evidence of multifocal disease suggestive of CNS demyelination. Ten patients with isolated IRV under the age of 50 had MRI of the brain (axial PD, T2, pre- and post Gadolinium) and optic nerves (coronal STIR). MRI was performed 2 to 12 years after the first manifestation of retinal disease.

Seven of 10 brain examinations were normal. 3 patients showed brain abnormalities: 1 had a single white matter lesion, 1 showed extensive white matter disease suggestive of 
ischaemia and 1 had multiple lesions indistinguishable from MS. After Gd-DTPA injection no pathological enhancement was observed. Only two patients showed abnormal high signal in the optic nerve, one of whom also had multiple white matter lesions. Our MRI findings support previous clinical data which show that the risk of developing MS with idiopathic retinal vasculitis is low. This contrasts sharply with clinical and MRI studies in isolated optic neuritis.

COMPARISON OF FAST SPIN ECHO (FSE) AND SHORT INVERSION TIME INVERSION RECOVERY (STIR) IMAGING IN OPTIC NEURTTIS (ON) A Gass, IF Moseley, D MacManus, DH Miller, WI McDonald. Institute of Neurology, London, UK

FSE is a new technique with scan times that are up to 16 times shorter than those obtained with conventional sequences, which allows very high resolution images of the optic nerve in 11 minutes. Optic nerve MRI was performed in 18 patients with $O N$ and in 10 normal controls. Two sequences were obtained coronally: FSE (in plane resolution $0.5 \mathrm{~mm}$, slice thickness $3 \mathrm{~mm}$ ) and STIR (in plane resolution $1 \mathrm{~mm}$, slice thickness $5 \mathrm{~mm}$ ), a sequence sensitive for demonstration of optic nerve lesions.

FSE demonstrated much more anatomical detail than STIR (for example, distinction of optic nerve and sheath). In ON, STIR and FSE were equally sensitive to lesion detection in 10 nerves, FSE appeared superior to STIR in 9 and only STIR detected a lesion in 2 cases. Two symptomatic nerves revealed no abnormality in both sequences. In several cases FSE alone demonstrated nerve swelling, atrophy or partial cross-sectional involvement of the nerves by lesions. In conclusion, FSE imaging of the optic nerve improves anatomical definition and increases lesion detection in ON.

CHRONIC INFLAMMATORY DEMYELINATING POLYNEUROPATHY MIMICKING A SPINAL STENOSIS SYNDROME

L Ginsberg, RHM King, J Workman, PK Thomas. Royal Free Hospital School of Medicine, London, UK

The authors report a patient with chronic inflammatory demyelinating polyneuropathy (CIDP) who developed cauda equina symptoms due to swelling of the nerve roots in the lumbar spinal canal. A 44 year old woman presented with a 13 year history of fluctuating back pain radiating to the legs exacerbated by exercise. She had mild distal sensory symptoms in the limbs. Examination revealed tendon areflexia and slight distal sensory loss. Electrodiagnostic studies indicated a demyelinating polyneuropathy. Sural nerve fascicular biopsy showed evidence of chronic demyelination with hypertrophic changes and the presence of inflammatory infiltrates. MRI of the lumbar spine revealed markedly thickened nerve roots from the level of the conus medullaris, filling the distal thecal sac. This case shows that spinal compressive syndromes may occur in acquired hypertrophic neuropathies as well as in hereditary motor and sensory neuropathy and expands the spectrum of the clinical presentation of CIDP.

ACCURACY, REPRODUCIBIITTY AND

VARIABILITY OF QUANTTTATIVE ASSESSMENTS OF BULBAR AND RESPIRATORY FUNCTION IN MOTOR NEURON DISEASE (MND)

A Goonetilleke, RJ Guiloff. Westminster Hospital, London, UK

Several drugs are being tested in MND. Survival studies require large numbers, are protracted and expensive. Another approach is to screen drugs eligible for survival studies by assessing deterioration of bulbar and respiratory function (which relate to survival in MND) over a period, in smaller samples. The validity of quantitative tests of these functions is studied.

Five raters timed repeated stimuli generated by a metronome simulating bulbar tests. For all raters, mean error-rate was $5 \cdot 1 \%$, correlation coefficient 0.89 and $\%$ difference 1.5 (readings 3 hours apart). Coefficient of variation (CV) of 10 readings was $3 \cdot 2 \%$.

The experienced rater obtained more reproducible and less variable readings, and performed timed bulbar (tongue, speech, jaw, swallowing) and respiratory tests (vital capacity, chest circumference) on 26 MND and 21 age and sex-matched controls over 5 days. Learning effects were eliminated. For all subjects and tests, mean correlation coefficient was 0.98 and $\%$ difference 2.3 (readings 3 hours apart), with CV $8 \cdot 8 \%$ (10 readings over 3 days); $M N D$ and control subjects behaved similarly. Composite bulbar scores improved reproducibility and variability. The estimated rater contribution to this variability was $17 \%$.

These tests are suitable for assessing deterioration of bulbar and respiratory function in small samples of MND patients.

ACCURACY, REPRODUCIBILITY AND VARIABILITY OF HAND-HELD DYNAMOMETRY IN MOTOR NEURON DISEASE (MND)

A Goonetilleke, RJ Guiloff. Westminster Hospital, London, UK

The limitations of manual testing of muscle strength are recognised. More objective and accurate methods are preferable in monitoring longitudinal changes, as required in clinical trials. The validity of readings obtained by hand-held dynamometry were investigated.

A spring-loaded device designed to "break" at preset forces was used to assess accuracy, reproducibility and variability of readings obtained by three raters with varying experience in the method. Accuracy (mean error-rate of $3.0 \%$ ), but not reproducibility or variability, was improved by greater experience.

The rater with intermediate experience tested strength in 9 muscle groups of 19 patients with MND. For all patients and muscles, mean correlation coefficient was 0.98 and $\%$ difference 13.2 (readings 6 days apart), with mean coefficient of variation (CV) of $9.9 \%$ (10 readings over 6 days). Use of a global composite muscle score resulted in improvements in reproducibility and variability, with mean correlation coefficient 0.98 (\% difference 6.7 ) and $\mathrm{CV}$
$5 \cdot 8 \%$. The rater was estimated to contribute $40 \%$ of the total variability of readings obtained whilst testing patients.

Hand-held dynamometry is a suitable method for assessing muscle strength in MND patients undergoing clinical trials.

MYORHYTHMIA AND OVERLAP SYNDROMES NJ Gutowski, JR Heron. North Staffordshire Royal Infirmary, UK

Five patients showing forms of an unusual tremor, myorhythmia, are presented. In all cases the palate and eye movements were not affected. The first two are cases of essential myorhythmia. The other cases show an overlap between myorhythmia and other tremors originating in the brainstem and cerebellar regions. Myorhythmia is a tremor which is present at rest and absent during sleep. It can be synchronous or asynchronous in different muscle groups and can have a variable rate in each muscle studied. It may be influenced by cough, stress, sustained posture, voluntary movement or peripheral stimulation. The patients presented demonstrate firstly that in essential cases of myorhythmia palatal tremor is absent. Secondly, that myorhythmia has a wide frequency range (at least 0.4 $\mathrm{Hz}$ to $6 \mathrm{~Hz}$ ). Thirdly, it can occur in association with other tremors originating in the brainstem, cerebellar and extrapyramidal pathways and fourthly there is commonly a familial history of tremor. To study the mechanisms underlying myorhythmia maximum information will be obtained from essential cases. It should be realised that myorhythmia can occur in the context of other tremors involving the brain stem, cerebellar and extrapyramidal circuits.

THE FUNCTIONAL LIMITATIONS PROFILE IS A VALID, RELIABLE AND SENSITTVE MEASURE OF DISABIILTY IN MULTIPLE SCLEROSIS

J Hutchinson, M Hutchinson. St Vincent's Hospital and University College, Dublin, Eire

The standard measure of disability in multiple sclerosis (MS), the Kurtzke Expanded Disability Status Scale (EDSS), is an ordinal scale subject to inter- and intrarater variability with a bimodal distribution in MS populations. The Illness Severity Score (ISS), is an interval score derived from the EDSS. The Functional Limitations Profile

(FLP) is a validated interval measure of patient report of disability.

On two occasions over six months 34 MS patients were assessed prospectively by a neurologist and a psychologist, using the above measures. The FLP Physical Dimension and its sub-scale Ambulation, correlated significantly with the EDSS for both visits (at visit $1: r=0.74$ and 0.81 respectively, at visit $2: r=0.76$ and 0.82 ). The reliability of FLP was assessed by comparing scores in the 23 patients who had not changed clinically (EDSS change $<1 \cdot 0$, ISS change $<3 \cdot 7$ ). The $99 \cdot 7 \%$ confidence limits (CL) were, for FLP Physical Dimension -4.5 to $+6 \cdot 1$, Ambulation $-8 \cdot 2$ to $+8 \cdot 7$. For sensitivity of the FLP the $11 \mathrm{MS}$ clinically changed patients (EDSS $\geqslant 1 \cdot 0$, ISS > 3.7) were assessed; $99.7 \% \mathrm{CL}$ were, for FLP Physical Dimension $-26 \cdot 3$ to $-0 \cdot 1$, Ambulation $-26 \cdot 5$ to +6.4 . 
The FLP in this pilot study is a valid, reliable and sensitive measure of MS disability. If confirmed by larger studies it would be useful in prospective studies of treatments of MS.

THE INFLUENCE OF HLA-DR AND -DQ ALLELES ON PROGRESSION TO MULTIPLE SCLEROSIS FOLLOWING A CLINICALLY ISOLATED LESION

MA Kelly, DA Cavan, MA Penny, $\mathrm{CH}$ Mijovic, D Jenkins, S Morrissey, DH Miller, AH Barnett, DA Francis. University of Birmingham and Institute of Neurology, London, UK

A five year follow up study was performed on 70 white patients presenting with isolated neurological syndromes of the optic nerve, brainstem or spinal cord to assess the risk of progression to multiple sclerosis (MS). The influence on patient prognosis of HLA-DR and -DQ alleles and presentation with disseminated brain lesions, demonstrated by MRI scanning, was determined. Clinical progression to MS was observed in $60 \%$ of optic neuritis patients, $50 \%$ of patients with a brainstem syndrome and $35 \%$ of patients with a spinal cord disturbance.

Multiple sclerosis and the isolated clinical syndromes were positively associated with DR15, DQA1*0102 and DQB1*0602-the frequency of the alleles in the latter group was intermediate between that seen in MS patients and healthy controls. Conversion to MS was positively associated with the DR15.DQA1*0102.DQB1*0602 haplotype but the influence of HLA was only apparent in patients with disseminated brain lesions at presentation (MRI-positive)-MS developed in $86 \%$ of MRI-positive, DR15-positive patients compared with $55 \%$ of MRI-positive, DR15-negative patients ( $p<$ $0.025)$. The data suggest that these HLA alleles are involved in susceptibility to initial demyelinating lesion formation and are important in the subsequent development of MS in MRI-positive patients.

BENIGN MS: MRI EVIDENCE FOR A LESS INFLAMMATORY DISEASE

D Kidd, AJ Thompson, DG MacManus, BE Kendall, WI McDonald. Institute of Neurology, London, UK

The development of disability in multiple sclerosis is highly variable, approximately one third of patients having a benign course. The reasons for this are not clear. Two longitudinal studies involving 11 patients with early relapsing/remitting disease and 11 with benign MS have been carried out. Patients had a clinical examination and a Gadolinium-DTPA enhanced MRI scan of brain at monthly intervals for six months. There was no difference in disability between the two groups. Mean MRI lesion loads were 41 and 48 respectively. Over the study period a mean of 0.5 relapses per relapsing/remitting patient and $0 \cdot 2$ relapses per benign patient occurred. There were 6.6 new lesions per relapsing/remitting patient and 1.9 new lesions per benign patient $(p<0.03)$. The number of new lesions per relapse was significantly less in the benign group $(2.25 \mathrm{v} 6.0, \mathrm{p}<0.03)$. Enhancement occurred in $80 \%$ and $38 \%$ of new lesions in relapsing/remitting and benign groups respectively.

Thus patients with benign MS appear to have fewer new MRI lesions per relapse and less evidence of blood-brain barrier breakdown and inflammation. This may result in less axonal damage and consequently less clinical disability.

INTEROBSERVER VARIATION IN THE

CLASSIFICATION OF SUBTYPES OF CEREBRAL INFARCTION

RI Lindley, MS Dennis, J Wardlaw, J Slattery, PAG Sandercock, CP Warlow. University of Edinburgh, UK

The classification of subtypes of cerebral infarction (total and partial anterior circulation infarction, lacunar infarction, and posterior circulation infarction) predicts: early mortality; functional outcome; and whether the infarct was due to large- or small-vessel occlusion. The reliability of the classification in clinical practice was examined.

Two clinicians independently assessed consecutive patients admitted to hospital with an acute stroke. Neurological history and signs, and the subtype of infarct were recorded.

Eighty five patients were assessed. Interobserver agreement for the classification was good (kappa 0.59; $95 \%$ confidence interval $0.43-0.74)$. Disagreement in the assessment of the commonly elicited neurological signs explained many of the misclassifications: interobserver agreement was good for some signs (hemiparesis kappa 0.77 , dysphasia 0.70 ), moderate for some (hemianopia 0.39 ) and poor for others (sensory loss $0 \cdot 15$ ).

The level of interobserver agreement is satisfactory. The classification can help identify priorities for investigation and research studies and is simple and practicable enough for use in routine clinical practice.

THE ANALYSIS OF THE PREDICTIVE LOCALISING VALUE OF CLINICAL SEIZURE PATTERNS DEFINED WITH THE ASSISTANCE OF CLUSTER ANALYSIS

M Manford, DR Fish, SD Shorvon Institute of Neurology and Charing Cross Hospital, London, UK

Localisation of clinical seizure patterns is currently defined by the "gold standard" of remission of seizures after resective surgery. This method is highly selective and introduces substantial bias. A novel approach is described.

A total of 252 cases were selected with focal abnormality of interictal EEG, ictal EEG, imaging or clinical pattern. Witnessed seizure histories were taken and sequence of seizure manifestations was encoded. Data were entered into a statistical cluster analysis and 25 groups identified, which were refined to 14 groupings by hand, each corresponding to a clinical seizure type. Location of lesions on imaging was analysed by a template technique and the involvement of different regions coded $0-3$. The relation between seizure type and regional cortical involvement was assessed, using chi-square analysis. Ictal and interictal EEG patterns were also assessed for each seizure type.
Well recognised seizure patterns were identified by this technique, for example, Jacksonian motor, which was strongly associated with perirolandic lesional involvement. Less familiar seizure types, for example, tonic posturing were also defined and this type was significantly associated with lateral premotor lesions $(p<0.001)$. In these groups, however, there were important minorities, with different associations on investigation. This analysis is less biased than in previous series and reflects the limitations of clinical seizure localisation.

EARLY RECOVERY SCALE FOR SEVERE TRAUMATIC BRAIN INJURY

DL McLellan, BA Wilson, S Horne, M Watson. Southampton University, UK

Chartering the recovery of patients with severe brain injury is difficult because current outcome scales are too crude. Eighty seven patients recovering from severe traumatic brain injury have been studied to establish the pattern and sequence of recovery of physical and psychological functions. The methodology required for this work is complex and will be discussed. An initial scale of 50 items has been identified covering the segment of recovery from coma to emergence from post-traumatic amnesia. These fifty items will be presented and show that the pattern of recovery is broadly similar to the sequence of normal functional development, but differs from it in some important respects. This scale and developments from it should prove helpful in the clinical assessment of patients and in defining objectives for rehabilitation.

A PROSPECTTVE STUDY OF THE CAUSES O SPASTIC PARAPARESIS AND QUADRIPARESIS IN 585 PATIENTS

AP Moore, LD Blumhardt. University of Liverpool, UK

In a three year period between 1986-89 a prospective study was carried out of the causes of non-traumatic spastic paraparesis and quadriparesis in a regional centre in the UK. A total of 585 patients were selected by intention to investigate by myelography. Patients under 15 years old or who had previously been investigated for myelopathy were excluded. One year after recruitment was halted the case notes were reviewed to ascertain the diagnosis.

The final diagnosis remained uncertain in $27.4 \%$, falling to $18.6 \%$ after MRI in a selected group. The commonest diagnoses were cervical spondylotic myelopathy $(23.6 \%)$, extrinsic tumour $(16.4 \%)$, multiple sclerosis $(9 \cdot 1 \%$ rising to $17 \cdot 8 \%$ after MRI) and motor neuron disease ( $4 \cdot 1 \%$ )

Ninety six patients had extrinsic compressive neoplasms, of which $41 \%$ were carcinomas, $20 \%$ were of nervous system origin, $17 \%$ haemopoeitic, $7 \%$ were cysts, $7 \%$ were mesenchymal. There were $41 / 585$ patients with previously known malignancy. In only 2 of these was an independent noncancerous pathology identified.

Reasons for an uncertain diagnosis included multiple pathology and insufficient evidence of causation. Others in this group may have multiple sclerosis or motorneurone disease. 
AMNESIC SYNDROME FOLLOWING

THEOPHYLIINE ASSOCIATED SEIZURES: IATROGENIC BRAIN INJURY

II O'Riordan, J Hutchinson, MX Fitzgerald, M Hutchinson. St Vincent's Hospital and University College, Dublin, Eire

Theophylline associated seizures usually occur with high theophylline serum levels and are often benign in nature. In some circumstances epileptic activity may be prolonged, focal in nature, resistant to therapy and with a poor outcome. The possibility that seizures associated with chronic theophylline therapy may cause brain injury is not generally recognised.

Two patients without a history of epilepsy, a 68 year old man with emphysema and a 16 year old girl with cystic fibrosis, developed status epilepticus when on chronic oral theophylline therapy. Although seizure activity was quickly controlled, both patients remained confused. Both have an amnesic syndrome confirmed by detailed neuropsychological testing. Anterograde amnesia persists six months after presentation and results in significant disability. MRI (T2 weighted) in one showed a high signal lesion in the left hypothalamus. Serum theophylline in one patient was in the mildly toxic range.

Theophylline associated seizures may result in severe brain injury and death. Various factors may predispose certain patients to develop seizures; these include advanced age, pre-existing neurological disease, low serum albumin and the concomitant use of cimetidine, ciprofloxacin and the erythromycin group of antibiotics.

SIDE OF NEUROLOGICAL SYMPTOMS PREDICTS RESULTS OF INVESTIGATION

PM Rothwell. Western General Hospital, Edinburgh, UK

Eighty two patients, under 60 years of age, who had undergone inpatient neurological investigation during the last 4 years, for unilateral sensory symptoms and/or weakness, in the absence of "hard" neurological signs, were identified by review of discharge summaries. Positive investigations, defined as those which explained the symptoms, were found in 22 cases [ $27 \%$ ]. Investigation was more frequently positive for right-sided than left-sided symptoms $[54 \%$ vs $13 \%, p<$ $0.01]$ and in males than females $[42 \% \mathrm{v}$ $19 \%, p=0.05$ ]. The number of investigations performed was not influenced by the side of symptoms or the sex of the patient (table).

Seventeen patients were described as anxious or hyperventilators at initial consultation, of whom 16 subsequently had negative investigations. Ten patients were seen by a psychiatrist during their in-patient stay and diagnosed to be depressed, of whom 9 had negative investigations. In patients with nor- mal investigations, anxiety was associated with left-sided symptoms [94\%] and depression with right-sided symptoms [89\%, p < 0.001].

In patients with unilateral symptoms and no "hard" signs the side of symptoms predicts the result of investigation. The opposite lateralisation of symptoms in anxiety and depression is consistent with experimental evidence of functional hemispheric asymmetry with altered mood.

FAMIIIAL BENIGN FASCICULATION

IK Saadeh, TK Banerjee, PJ Hughes, LS Illis. Wessex Neurological Centre, Southampton, UK

Fasciculations may occur as part of a benign syndrome. Fasciculations in 3 family members are described. The father and elder son were symptomatic, whereas the younger son was asymptomatic. The authors have been unable to find any previous report of several affected members within one family.

Patients with fasciculations or muscle cramps without the presence of wasting or weakness or EMG evidence of denervation are unlikely to develop motor neuron disease. Fasciculations, have been described in otherwise normal individuals, peripheral neuropathies and a variety of toxic, drug induced and metabolic disturbances. Differentiation between malignant and benign fasciculation of healthy people remains uncertain

Electrophysiological findings are probably not helpful in distinguishing between malignant and benign fasciculation, although some differences have been described. Although 3 cases of benign fasciculation occurring in 2 generations of one family have been identified, the pathogenesis of this disorder is uncertain.

POSSIBLE PREFERENTIAL EFFECT OF DEMYELINATION ON THE MAGNOCELLULAR PATHWAY

R Sehdev, DH Foster, JR Heron. University of Keele and North Staffordshire Royal Infirmary, UK

Psychophysical investigation of patients with optic neuritis and multiple sclerosis has revealed conflicting evidence supporting selective and non-selective losses in various components (spatial, temporal, luminance and colour) of visual function. On the basis of anatomical, physiological, and psychophysical studies in humans and other primates, two major pathways of visual processing have been identified, the magnocellular pathway and the parvocellular pathway. These pathways may provide a framework for explaining the different patterns of visual loss in demyelinating disease.
Measurements were made in a group of six patients with previous optic neuritis with relatively mild residual impairment following their last episode of optic neuritis, and in a group of five healthy, age- and sexmatched controls. Three kinds of flickering stimuli, with different spatial configurations, were used to obtain a measure of temporal modulation sensitivity at different temporal frequencies, in the form of de Lange attenuation characteristics. Patients showed losses in modulation sensitivity in all conditions, but most strongly for stimuli with relatively low effective spatial frequency, independent of temporal frequency, and for one condition of relatively high spatial frequency. A possible interpretation of these findings is that there is a preferential effect of demyelination on the magnocellular pathway.

NON-VACUOLAR MYELOPATHIES IN HIV INFECTION

SV Tan, RJ Guiloff, F Scaravilli, N Harcourt-Webster, BG Gazzard Westminster Hospital and Institute of Neurology, London UK

Non-vacuolar myelopathies in HIV infection have not been well documented. Thirty eight patients with myelopathy seen over eighteen months were drawn from a population of 488 patients with AIDS, 1015 symptomatic non-AIDS/PGL, 407 well seropositives, 10 with seroconversion illness, and 21 of undetermined status.

Eighteen patients had clinical, radiological and/or pathological data incompatible with a diagnosis of vacuolar myelopathy (VM). All were male homosexuals; median age 34 years (range 21-61), median CD4 count 49 cells $/ \mathrm{ml},(2-520)$. Seven had acute myelopathies (H.zoster 2, probable CMV 2 , extradural lymphomatous deposits 1 , cord contusion 1, undetermined 1). Five had acute myeloradiculopathies (H.zoster 1, CMV 4). Four ${ }^{\star}$ had chronic myelopathies (cervical spondylosis 2, undetermined 2). One, with intellectual impairment and myelopathy mimicking VM, recovered spontaneously. One had a hysterical paraparesis. The diagnosis was confirmed pathologically in 2 (H.zoster myelitis, CMV radiculomyelitis).

The annual incidence of non-vacuolar myelopathies in our population was $1.4 \%$ in AIDS, $\quad 0.5 \%$ in symptomatic nonAIDS/PGL, and $0 \cdot 2 \%$ in well seropositives. Potentially treatable causes included cervical spondylosis, CMV myelitis/myeloradiculitis and H.zoster myelitis. Subacute/chronic myelopathies should not be diagnosed as VM without adequate investigation. CMV infection was common in acute myelopathy/myeloradiculopathy; concomitant retinitis should be sought.

*One had a subacute myeloradiculopathy (CHV).

HERPES SIMPLEX TYPE-I ENCEPHALITIS IN AIDS

SV Tan, RJ Guiloff, F Scaravilli, PE Klapper, GM Cleator, BG Gazzard. Westminster Hospital and Institute of Neurology, London and University of Manchester UK

Classical HSV-1 encephalitis has not been described in AIDS. It has been suggested that severely immunocompromised AIDS patients may be unable to mount the necro- 
tising immune reaction responsible for the classical clinicopathological picture.

An AIDS patient is presented with a CD4 count of 161 cells $/ \mu$ l and clinically typical acute HSV-1 encephalitis with severe cerebral necrosis in the distribution seen in immunocompetent subjects. CT scan and EEG showed bitemporal abnormalities. Serum viral titres, CSF antigenspecific oligoclonal studies and PCR for HSV were negative. He died 4 weeks from the onset of illness.

There was swelling of both temporal lobes, bilateral uncal herniation and bilateral extensive necrotic change in the hippocampi, insulae, and parahippocampal and cingulate gyri. Unlike HSV encephalitis in immunocompetent patients, where viral antigen is rarely demonstrable after 3 weeks of illness or after antiviral treatment, viral inclusions were abundant and easily visible on $\mathrm{H}+\mathrm{E}$ and with immunostaining, as described in one previous anergic non-HIV patient. PCR of brain tissue identified the virus as HSV-1.

The severe acute necrotising immune reaction appeared to be largely macrophage and/or monokine mediated; poor viral clearance may have been due to impaired T-cell function.

SERUM OLIGOCLONAL BANDS IN MULTIPLE SCLEROSIS

AZJ Zeman, G Keir, EJ Thompson, WI
McDonald. Institute of Neurology, London, UK

The detection of oligoclonal bands of IgG in CSF, in their absence from serum, has proved a robust and sensitive method for demonstrating an intrathecal immune response in the investigation of multiple sclerosis. The presence of oligoclonal bands in the serum in multiple sclerosis has been noted in the past, but there has been considerable disagreement over their incidence and significance. We have examined CSF and serum pairs from 50 consecutive patients with CSF oligoclonal bands, undergoing investigation for multiple sclerosis, using the sensitive technique of isoelectric focusing followed by immunodetection. Serum oligoclonal bands were present in $46 \%$ of cases. There are both: 1) qualitative and 2) quantitative reasons for concluding that these oligoclonal bands reflect systemic synthesis of oligoclonal IgG which is independent of the intrathecal immune response: 1) the serum pattern is discordant with the CSF pattern in $87 \%$ of patients with serum oligoclonal bands, indicating independent synthesis in the two compartments; 2) quantitative measurement of intrathecal IgG production in these patients, combined with dilution experiments, indicate that clones synthesised intrathecally will very rarely achieve a sufficient concentration in serum to be detected. These findings support the view that systemic immune activation is common in multiple sclerosis.
OLIGOCLONAL BAND NEGATIVE MULTIPLE SCLEROSIS

AZJ Zeman, D Kidd, B McLean, E Thompson, WI McDonald. Institute of Neurology, London, UK

The demonstration of intrathecal synthesis of oligoclonal IgG has diagnostic and prognostic implications in suspected multiple sclerosis, and the oligoclonal IgG may play a part in the pathogenesis of the disease. Oligoclonal bands are absent from about $10 \%$ of cases of clinically definite multiple sclerosis, but this group of patients has seldom been studied. Twenty five such patients have been identified among 247 patients with clinically definite multiple sclerosis $(10 \cdot 1 \%)$. On detailed review of these 25 cases the laboratory results proved to be equivocal or technically unsatisfactory in 12 $(48 \%)$, and 5 patients had plausible alternative explanations for their symptoms (25\%). Ten oligoclonal band negative patients with unequivocal laboratory results and clinical diagnosis were matched for age, sex, disease duration and type with 10 oligoclonal band positive controls. Disability was assessed on the Kurtzke scale by an examiner blind to the CSF findings. In 7 of the 10 pairs the positive control was more severely disabled, while in only 2 pairs was the negative patien more severely affected $(p=0 \cdot 01)$. Absence of oligoclonal bands in putative clinically definite multiple sclerosis should raise suspicion of misdiagnosis or laboratory error, but undoubted cases of oligoclonal and negative multiple sclerosis do occur, and may have a relatively benign prognosis. 